\title{
Thoracic lymphadenopathy in Asians resident in the United Kingdom: role of mediastinoscopy in initial diagnosis
}

\author{
PR FARROW, DA JONES, PJ STANLEY, JS BAILEY, JM WALES, JB COOKSON \\ From the Regional Cardiothoracic Centre, Groby Road Hospital, Leicester
}

\begin{abstract}
Although the incidence of superficial glandular tuberculosis is high in Asian immigrants, a clinical diagnosis without biopsy has previously been shown to be inaccurate in $22 \%$ of cases. The role of diagnostic mediastinoscopy and biopsy in thoracic lymphadenopathy was therefore evaluated in 41 consecutive Asian patients. Tuberculosis was diagnosed by histological examination or culture (or both) of gland biopsy material in 24 (59\%). A further 12 patients, however, also received antituberculous chemotherapy, with a response in 10 cases. If these are included, $34(83 \%)$ were finally considered to have tuberculosis. The symptoms and ages of the patients with tuberculosis were similar to those typically seen in caucasians with sarcoidosis. Six had tuberculous bilateral hilar lymphadenopathy. In only four cases $(10 \%)$ was a positive diagnosis other than tuberculosis established; in two (lymphoma and thymoma) mediastinoscopy confirmed preoperative suspicions, and saved only two more (with sarcoidosis and vascular anomaly) from unnecessary antituberculous treatment. Complications included severe haemorrhage (1) and chronic tuberculous sinus in the endoscopy tract (2). Mediastinoscopy is unlikely to change management in most patients, produces an appreciable amount of morbidity, and should be reserved for cases in which there is additional clinical doubt. Any future decline in the prevalence of tuberculosis in Asians may, however, require its further evaluation.
\end{abstract}

Tuberculous lymphadenitis is common in Britain among immigrants of Asian origin and in a recent national survey accounted for about $40 \%$ of the cases of tuberculosis notified in this ethnic group. ${ }^{1}$ Because of this incidence persistent glandular enlargement in Asians has often been diagnosed and treated presumptively as tuberculosis. Histological confirmation is reserved for patients who fail to respond. This approach, however, may delay the diagnosis of other treatable conditions, such as lymphoma, which may be indistinguishable clinically. After such a case had been seen in this unit we decided to perform biopsies on all enlarged glands in Asian patients. We have previously reported that biopsy of superficial glands thought to be tuberculous yielded an alternative diagnosis in $22 \%$ of cases and was a worthwhile procedure. ${ }^{2}$ We now evaluate

Address for reprint requests: Dr PR Farrow, Department of Medicine, Glenfield General Hospital, Groby Road, Leicester LE3 9QP.

Accepted 3 October 1984 the role of mediastinoscopy in the diagnosis of enlarged intrathoracic glands in Asians.

\section{Patients and methods}

Forty two consecutive Asian patients underwent mediastinoscopy in a regional cardiothoracic unit from 1977 to 1982 . Data were extracted from case records. The mean age was 37 (range 14-68) years and 27 were male. Most had immigrated from the Indian province of Gujarat either directly or via East Africa.

One patient with proved bronchial carcinoma was submitted to mediastinoscopy as a preoperative staging procedure and has been excluded from further analysis. In the remaining 41 the investigation was performed for diagnosis. All patients had radiological evidence of mediastinal gland enlargement (table 1) but none had more accessible lymphadenopathy or overt pulmonary disease. Thirty six underwent mediastinoscopy before antituberculous chemotherapy and five were referred from 
Table 1 Radiological distribution of thoracic gland enlargement in 41 Asians undergoing diagnostic mediastinoscopy

\begin{tabular}{lc}
\hline Site & No of cases \\
\hline Right paratracheal & 32 \\
Left paratracheal & 7 \\
Hilar & ${ }^{*}$ \\
Right & 11 \\
Left & 9 \\
\hline
\end{tabular}

*Seven patients had bilateral hilar lymphadenopathy, which in six cases was tuberculous ( 16 patients had two or more affected sites.)

other physicians after an apparent failure to respond to antituberculous treatment. Mediastinoscopy was performed under general anaesthesia after the method of Carlens, ${ }^{3}$ except that streptomycin was not inserted into the mediastinoscopy tract. Biopsy specimens of lymph nodes were taken whenever possible.

In an attempt to quantify the response to antituberculous chemotherapy, where this was given, serial measurements of gland diameter were made from all available posteroanterior chest radiographs by two of us (PRF and DAJ). Patients were classified as responders if either the chest radiograph had returned to normal or there was at least $50 \%$ reduction in gland diameter and no relapse after completion of treatment.

\section{Results}

Cough was the most common presenting symptom, occurring in $68 \%$ of cases, with weight loss in $51 \%$ and fever or sweats in $46 \%$. An enlarged liver or spleen was found in $12 \%$, all of whom had tuberculosis as the final diagnosis.

Biopsy material was obtained from 39 patients. In one of the remaining two patients no lymph nodes were found, and in the other, a 44 year old man, a thymoma was visualised but no biopsy specimen was taken. Results of mediastinoscopy are summarised in table 2.

Tuberculosis was confirmed by histological examination or culture (or both) in 24 patients

Table 2 Mediastinoscopy results and radiological response to antituberculous chemotherapy in 41 Asians with thoracic lymphadenopathy

\begin{tabular}{llll}
\hline Mediastinoscopy result & $\begin{array}{l}\text { Total } \\
\text { No }\end{array}$ & $\begin{array}{l}\text { No } \\
\text { treated }\end{array}$ & $\begin{array}{l}\text { No of } \\
\text { responders }\end{array}$ \\
\hline Tuberculosis & 24 & 24 & 22 \\
Sarcoidosis & 7 & 6 & 4 \\
Non-specific or normal & 7 & 6 & 6 \\
Thymoma & 1 & & \\
Lymphoma & 1 & & \\
No lymphoid tissue found & 1 & & \\
\hline
\end{tabular}

(59\%). Histological examination showed tuberculosis (caseating granulomas with or without giant cells or the presence of alcohol and acid fast staining bacilli) in 20 of the 24 biopsy specimens examined in this group; of the 18 cultures, 14 grew Mycobacterium tuberculosis var hominis and four were culture negative. No mycobacterial cultures grew atypical variants and all were reported as sensitive to standard antituberculous agents.

Of the remaining 15 patients from whom biopsy material was obtained, seven showed non-caseating granulomas; the five cases in which culture was performed were negative. Six of the seven, however, were treated presumptively for tuberculosis either because of clinical doubt or a positive tuberculin test. Four of these six apparently responded and two had persistent gland enlargement after treatment but without other clinical evidence of active disease. The remaining patient with non-caseating granulomas and a grade 3 Heaf test later developed pulmonary sarcoidosis, which cleared with steroid treatment alone.

The biopsy specimens from seven patients failed to establish any firm diagnosis, having either nonspecific or normal histological appearances or tissue which was not lymph node. In one patient with normal histological appearances mediastinoscopy showed a vascular anomaly affecting the innominate artery as the cause of the radiological abnormality. Tissue was cultured in six cases-all with negative results. Six of the seven patients with a nondiagnostic finding at mediastinoscopy responded radiologically to a trial of antituberculous chemotherapy.

The biopsy material from the 15 th patient (a 14 year old girl) showed Hodgkin's lymphoma.

Of the five patients referred for biopsy after apparent failure of treatment, mediastinoscopy confirmed tuberculous histological appearances in two and showed non-caseating granulomas in two (one of whom subsequently responded to antituberculous treatment) and non-specific histological appearances in one with no further gland regression occurring with continued treatment. All cultures performed in this group were negative.

The results of tuberculin testing were available for 36 patients. One negative result ( $\leqslant$ grade 1 Heaf test) occurred in the tuberculosis group and there were two positive results ( $\leqslant$ grade 3 Heaf test) in patients who did not have tuberculosis. $M$ tuberculosis was cultured from sputum or laryngeal swabs from two patients, both of whom had positive cultures of mediastinal glands.

Morbidity produced by mediastinoscopy included one serious haemorrhage in a 35 year old woman, which required thoracotomy for control. Biopsy 
confirmed tuberculosis. Two patients, also with tuberculosis, developed chronic sinuses in the mediastinoscopy tract, which discharged for four and 10 weeks despite appropriate chemotherapy. Pain requiring analgesia longer than 24 hours after operation was present in $20 \%$ of patients and the average length of stay for mediastinoscopy was 4.5 days.

The median length of antituberculous chemotherapy was 12 months (range 9-24 months); variations can be ascribed to the practice of individual physicians and, in one case (where biopsy had been performed after treatment had started), to doubts about compliance. All 36 patients treated for tuberculosis received isoniazid and rifampicin. For the first two months 34 patients also received ethambutol and two received streptomycin.

In the group classified as responders to antituberculous treatment, the median duration of treatment up to the last recorded abnormal chest radiograph was 4.4 months (and up to the first radiograph considered normal 8.2 months). In only one patient, who had culture positive tuberculosis, was regression in gland size still seen after nine months of treatment and in no series of radiographs was there evidence of further gland enlargement after the start of treatment.

\section{Discussion}

The diagnosis of thoracic gland enlargement can be difficult and often based on probabilities. ${ }^{4}$ Bilateral hilar lymphadenopathy in caucasians is usually due to sarcoidosis (74\%), lymphoma accounting for only $20 \%$ of cases and tuberculosis being uncommon. ${ }^{5}$ In our series of Asian patients, who were unselected by final diagnosis, tuberculosis was confirmed by either histological examination or culture of biopsy material in $59 \%$. If those whose biopsy gave a negative result but who nevertheless received antituberculous chemotherapy and had regression of glands are included, the overall incidence of tuberculosis was $83 \%$.

The clinical symptoms in the tuberculosis group were similar to those reported in sarcoidosis, ${ }^{5}$ but affected a higher percentage of patients. Most, however, had remained relatively well, the common presenting symptoms being cough, minor degrees of weight loss, and fever. Radiological findings also overlap. Bilateral tracheobronchial glands were seen in $32 \%$ and in particular bilateral hilar lymphadenopathy occurred in $18 \%$ of patients with tuberculosis; thus it is not a rarity as has been previously suggested. ${ }^{6}$

Mediastinoscopy is an established method of diagnosing tuberculous thoracic lymphadenitis, dis- cussed in several reports ${ }^{7-10}$; but these have not prospectively evaluated its use in Asians, in whom the incidence of tuberculosis is high. In our series mediastinoscopy successfully diagnosed only $71 \%$ of cases finally considered to have been tuberculous and one sixth of these positive results were from cultures available only after necessary decisions about treatment had been taken.

Four patients $(10 \%)$ had diagnoses other than tuberculosis made as a result of mediastinoscopy. The patient with lymphoma was the youngest (age 14), had been particularly ill, and had a negative reaction to the tuberculin test. Glandular tuberculosis is commonest in early middle age and a clinical diagnosis in the young or elderly should be made with caution. The patient with a thymoma also was tuberculin negative. Thus in these two cases there were grounds to suspect a diagnosis other than tuberculosis. In contrast, the two with sarcoidosis and vascular anomaly both had positive reactions to the Heaf test and if mediastinoscopy had not been performed they would have been treated inappropriately as having tuberculosis. Nevertheless, apart from possible drug toxicity, the eventual outcome probably would have been no different.

The major adverse effects of mediastinoscopy, haemorrhage and chronic sinus formation, occurred only in the patients with tuberculosis. Haemorrhage occurs in $0.5 \%{ }^{11}$ to $1.8 \%^{12}$ of all mediastinoscopies in thoracic units but a higher incidence is likely if a diagnostic biopsy is attempted at every procedure as in our patients. Implantation of tumour very occasionally follows mediastinoscopy ${ }^{13}$ but there is no report of tuberculous sinuses in several large reviews. ${ }^{11} 1415$ In our two patients there was no delay in starting treatment but both had extensive mediastinal disease and one, who had associated pericarditis, also received steroids, which may have delayed wound healing. The occurrence is perhaps not unexpected as scar breakdown and sinus formation may follow up to $10 \%$ of operations on superficial tuberculous glands despite appropriate chemotherapy. ${ }^{16}$

We conclude that obtaining tissue from enlarged thoracic glands is unlikely to change the management of most Asians, causes infrequent but important complications, and is unnecessary as a routine. To provide criteria for performing diagnostic mediastinoscopy it is suggested that patients should (1) be young, (2) have a negative tuberculin test reaction, (3) have atypical clinical or haematological features, or (4) have further gland enlargement after commencement of treatment. Such an approach would have limited mediastinoscopy to $12 \%$ and led to unnecessary treatment in only $5 \%$ of our patients.

Most glandular tuberculosis in immigrants occurs within five years of arrival in Britain. ${ }^{17}{ }^{18}$ As the last 
substantial increase in residents of Asian origin was in the earlier part of the last decade, the incidence of tuberculous mediastinal lymphadenitis is likely to fall and the proportion of cases with a nontuberculous cause of thoracic gland enlargement to rise. These altered incidences may then make clinical diagnoses unsupported by biopsy less accurate.

\section{References}

1 Medical Research Council Tuberculosis and Chest Diseases Unit. National survey of tuberculosis notifications in England and Wales 1978-9. Br Med J 1980;281:895-8.

2 Stanley P, Farrow PR, Jones DA, Cookson JB, Wales JM. Glandular tuberculosis in Asians - is clinical diagnosis sufficient? Thorax 1981;36:711 (abstract).

3 Carlens E. Mediastinoscopy: a method for inspection and tissue biopsy in the superior mediastinum. Dis Chest 1959;36:343-52.

4 Scadding JG. Sarcoidosis, London: , Eyre and Spottiswoode, 1967:496.

5 Winterbauer RH, Belic N, Moores DM. A clinical interpretation of bilateral hilar adenopathy. Ann Intern Med 1973;78:65-71.

6 Sakowitz AJ, Sakowitz BH. Bilateral hilar lymphadenopathy: an uncommon manifestation of adult tuberculosis. Chest 1977;71:421-3.
7 Carlens E, Herlitz L. Mediastinoscopy as an aid in the diagnosis of intrathoracic tuberculosis. Acta Tuberc Scand 1964;35:35-40.

8 Lincoln JCR, Provan JL. Mediastinoscopy in the diagnosis of non-malignant thoracic disease.J Thorac Cardiovasc Surg 1970;60:144-8.

9 Mikhail JR, Mitchell DN. Mediastinoscopy: a diagnostic procedure in hilar and paratracheal lymphadenopathy. Postgrad Med J 1971;47:698-704.

10 Cameron EWJ. Tuberculosis and mediastinoscopy. Thorax 1978;33:117-20.

11 Lee CM, Grossman LB. Complications associated with mediastinoscopy. Penn Med 1977;80:39-42.

12 Doctor AH. Mediastinoscopy: a critical review of 220 cases. Ann Surg 1971;174:965-8.

13 Barraclough BH, Richards HJ, Morck I. Mediastinoscopy: a safe and useful procedure in the investigation of patients with intrathoracic diseases. Aust NZ J Surg 1971;41:153-7.

14 van der Schaar PJ, van Zanten ME. Experience with mediastinoscopy. Thorax 1965;20:211-3.

15 Kliems G, Savic B. Complications of mediastinoscopy. Endoscopy 1979;1:9-12.

16 Campbell IA, Dyson AJ. Lymph node tuberculosis: a comparison of various methods of treatment. Tubercle 1977; 58:171-9.

17 British Thoracic Association. Tuberculosis among immigrants related to length of residence in England and Wales. Br Med J 1975;iii:698-9.

18 Summers GD, McNicol MW. Tuberculosis of superficial lymph nodes. Br J Dis Chest 1980;74:369-73. 